\title{
Positional changes in tendon insertions from bone to fascia: development of the pes anserinus and semimembranosus muscle insertion in human foetuses
}

\author{
Z.W. Jin ${ }^{1}$, H. Abe ${ }^{2}$, Y. Jin ${ }^{1}$, S. Shibata ${ }^{3}$, G. Murakami ${ }^{4}$, J.F. Rodríguez-Vázquez ${ }^{5}$ \\ ${ }^{1}$ Department of Anatomy, Histology and Embryology, Yanbian University Medical College, Yanbian, China \\ 2Department of Anatomy, Akita University School of Medicine, Akita, Japan \\ ${ }^{3}$ Department of Maxillofacial Anatomy, Graduate School of Tokyo Medical and Dental University, Tokyo, Japan \\ ${ }^{4}$ Division of Internal Medicine, Iwamizawa Kojin-kai Hospital, Iwamizawa, Japan \\ ${ }^{5}$ Department of Anatomy and Human Embryology, Institute of Embryology, Faculty of Medicine, Madrid, Spain
}

[Received: 3 February 2016; Accepted: 4 March 2016]

Development of a long muscle belly in foetal extremities generally requires a definite bony insertion of the long tendon. However, in adults, the pes anserinus and the semimembranosus tendon (SMT) are inserted into fasciae. Development of fascial insertions in foetuses was investigated by examining serial histological sections obtained from 7 foetuses at 8-9 weeks and 8 foetuses at 14-16 weeks. The presence of matrix substances and macrophages was also examined by immunohistochemistry. At 8 weeks, the tendons of the semitendinosus, gracilis, sartorius and semimembranosus muscles were straight and inserted into the initial shaft-like proximal end of the tibia on the proximal side of the popliteus muscle. At 9 weeks, however, the medially extending popliteus muscle appeared to push the pes anserinus tendons superficially, with a loss of cartilage insertions. The SMT obtained an attachment to the popliteus muscle. At 14-16 weeks, the SMT divided into thick and thin bundles: the former contained abundant macrophages and inserted into the tenascin-positive perichondrium of the enlarged proximal tibia, while the later without macrophages ended at the joint capsule. The pes anserinus tendons, negative for both versican and tenascin-c, took highly tortuous courses toward the fascia cruris. Because the medial extension of the popliteus muscle was associated with the enlargement of the proximal tibia, the topographical relationship of the popliteus muscle with these 4 tendons changed drastically, resulting in a loss of cartilage insertion of the pes anserinus tendons as well as the division and reconstruction of the SMT. (Folia Morphol 2016; 75, 4: 503-511)

Key words: pes anserinus, sartorius muscle, gracilis muscle, semimembranosus tendon, popliteus muscle, human foetuses

\section{INTRODUCTION}

The tendons of the semitendinosus, gracilis and sartorius muscles join together to form a common tendinous tissue, the pes anserinus, which is inserted into the fascia cruris [15]. These tendons in the pes anserinus form layers, from the superficial sartorius to the deep semitendinosus tendon [11]. Despite the absence of definitive bony insertion into the tibia,

Address for correspondence: Z.W. Jin, MD, PhD, Department of Anatomy, Histology and Embryology, Yanbian University Medical College, Yanji City, Jilin Province, 133002, China, tel: +86-433-243-5115, fax: +86-433-243-5103, e-mail: zwjin@ybu.edu.cn 
each of these muscles has a long muscle belly. Near the pes anserinus, the semimembranosus muscle shows an unusual insertion, with half of the tendon fibres merging with a thick aponeurosis covering the popliteus muscle and the other half directed upward and continued to the oblique popliteal ligament [15]. The morphologies of these muscle insertions at the medial side of the knee differ markedly from the morphologies of lateral side muscles, such as the biceps femoris, which has a definite tendinous insertion into the fibular head. To our knowledge, however, little is known about the development of the pes anserinus and the semimembranosus muscle insertion in human foetuses. The morphology of the medial aspect of the foetal knee is also unusual, in that the semimembranosus and semitendinosus tendons provide deep notches on the gastrocnemius muscle belly for crossing [14].

To date, no tendon in human foetal extremities has been reported to show a highly tortuous course near the bony insertion, as shown for the shoulder and arm [1], forearm and hand [7], and foot and ankle [21]. Conversely, foetal development of a long muscle belly may require a short or long straight tendon attached to the hard tissue. Mechanical loading is required for tendon regeneration and repair $[8,22]$. Similarly, dynamic loading by skeletal muscles is critically important for the morphogenesis of tendon and its bony insertions. During foetal development, early muscle contraction confers a specific morphology on a tendon $[8,9]$, with the tendon conferring a specific shape on the bone $[16,18]$. Following shortening of a tendon in adult rabbits, tendon reconstruction occurred 7 weeks after muscle fibre adaptation [20]. Although many studies have investigated the molecular basis of tendon differentiation $[2,12,17]$, no researchers paid attention to the foetal development of a fascial insertion of the tendon. Examination of serial sections of human embryos showed sequential changes in tendon insertion sites for the posterior bellies of the digastricus muscle [6] and for the obturator internus muscle [13]. Changes in topographical anatomy seemed to promote reconstruction of the tendon insertion. This study hypothesized that, during foetal development of the pes anserinus and semimembranosus tendon (SMT), insertion sites change, from a hard tissue to a fascia. This study was performed to describe early foetal development of tendon insertions on the medial side of the knee.

\section{MATERIALS AND METHODS}

The study was performed in accordance with the provisions of the Declaration of Helsinki 1995 (as revised in 2013). Sagittal serial sections were obtained from 15 human foetuses: 7 of gestational age 8-9 weeks (crown-rump length [CRL] 25-38 mm) and 8 of gestational age 14-16 weeks (CRL 115-155 mm); the latter included 2 foetuses aged 14 weeks; 4 aged 15 weeks; and 2 aged 16 weeks.

The sections of the 7 foetuses aged 8-9 weeks were parts of the large collection kept at the Institute of Embryology, Universidad Complutense Madrid, and were products of miscarriages and ectopic pregnancies managed at the Department of Obstetrics at the University. These sections in Madrid, 5-8 micron in thickness, had been stained with haematoxylin and eosin (HE), azan or silver staining. The sectional planes were sagittal to the body, and each section contained one or both extremities. The use of these sections was approved by the University Ethics Committee in Madrid (No. B08/374).

The 8 foetuses of gestational age 14-16 weeks were parts of a foetus collection kept by Department of Anatomy, Akita University, Akita, Japan. These foetuses had been donated by their families to the Department in $1975-1985$, fixed by immersion in $10 \% \mathrm{v} / \mathrm{v}$ neutral formalin solution and stored in $50-70 \% \mathrm{v} / \mathrm{v}$ ethanol solution for more than 30 years. The available data was limited to the date of donation and the gestational age. There were no documents reporting family name, the names of obstetricians and hospitals and the reasons for abortion. The use of these foetuses for research was approved by the Akita University Ethics Committee (No. 1428). After separating the lower extremities from the body of each foetus stored at Akita University, the knees were decalcified by incubation at $4^{\circ} \mathrm{C}$ in 0.5-mol/LEDTA (pH 7.5) solution (Decalcifying Solution B; Wako, Tokyo, Japan) for 3-5 days, depending on the size of the sample. Paraffin blocks were obtained from 12 knees of the 8 foetuses in Akita. Each knee was accompanied by the distal thigh and proximal leg. Each block was used to prepare 80-120 serial sagittal sections covering the medial half of the knee ( 5 microns thick; 20-50 micron intervals). Every fifth section was stained with $\mathrm{HE}$, while the other four sections were used for immunohistochemistry.

For immunohistochemistry, the sections were pretreated with testicular hyaluronidase $(25 \mathrm{mg} / \mathrm{mL}$; Sigma type I-S; Sigma Chemicals, St. Louis, MO, USA) in phosphate-buffered saline for $30 \mathrm{~min}$ at $37^{\circ} \mathrm{C}$ to 
identify the two matrix substances [19]. The sections were subsequently incubated with mouse monoclonal anti-versican core protein antibody (12C5; Developmental Studies Hybridoma Bank), diluted 1:25; rabbit polyclonal anti-rat tenascin-c antibody (Chemicon, Temecula, CA, USA), diluted 1:100; and mouse monoclonal anti-human CD68 KP1 antibody (Dako M0814), diluted 1:100. After washing, the sections were incubated for $30 \mathrm{~min}$ in Histofine Simple Stain Max-PO (Nichirei, Tokyo, Japan), followed by treatment with diaminobenzidine (DAB) and horseradish peroxidase (HRP). Each experiment included a negative control, consisting of tissue specimens without primary antibody. Sections stained for CD68 were counterstained with haematoxylin. Results were observed and photographed with a Nikon Eclipse 80 microscope.

\section{RESULTS}

The topographical relationships of muscles differed between foetal stages. Sections of specimens at 14-16 weeks were prepared sagittally to the distal thigh and proximal leg, whereas sections of specimens at 8-9 weeks were sagittal to the body, not to the lower extremity. Because rotation at the hip joint was lower in the earlier sections, the legs were directed more posteriorly. Thus, at 8-9 weeks, a single plane showed the posterior thigh muscles (e.g. the semimembranosus and semitendinosus muscles) together with the anteromedial muscles (e.g. the gracilis and sartorius muscles). In sections of the later specimens, as in adults, the semimembranosus muscle belly was observed on the lateral side of the gracilis and sartorius muscles.

\section{Foetuses of gestational age 8-9 weeks}

The joint cavity and meniscus were not seen in the putative knee joints of the 3 smallest specimens, of CRL 25, 28 and $29 \mathrm{~mm}$ and gestational age approximately 8 weeks (Fig. 1). The fibular head was $0.5-1.0 \mathrm{~mm}$ distal to the knee joint. The tibial plateau and bilateral condyles (a wide proximal end with a reversed triangle shape) had not yet formed, giving the proximal tibia a shaft-like appearance. The popliteus muscle was located near the distallylocated fibular head, but the popliteus tendon and its attachment to the femur were not observed. Notably, the future pes anserinus tendons (tendons of the semitendinosus, gracilis and sartorius muscles) approached the initial shaft-like tibia and appeared to be inserted into the perichondrium of the far proximal side of the popliteus muscle (Fig. 1A). The SMT ended in a dense tissue along the tibia near the knee joint. The sites of insertion of the sartorius and semitendinosus tendons were located on the immediately distal side of the SMT terminal (Fig. 1C, D). The gracilis tendon, the most medially located of these 4 tendons, appeared to run alongside or wind around the perichondrium (Fig. 1E, F). The fascia cruris was not yet developed. A notch was not present on the medial head of the gastrocnemius muscle.

The proximo-distal length from the knee joint to the fibular head was not different between the 4 specimens of CRL 30-38 mm (approximately 9 weeks) and the aforementioned 3 smaller specimens. However, the lateral-medial distance (or intervals between panels in figures) was more than 2-fold greater in the 4 larger than in the 3 smaller specimens. The tibial condyles were developing. The meniscus was present, but the joint cavity at the putative knee was unclear (Fig. 2). The popliteus muscle extended alongside the enlarging posterior aspect of the proximal tibia. The initial pes anserinus was observed on the immediately medial side of the knee joint, despite the absence of a definite fascia cruris (Fig. 2E-G). A thick portion of the sartorius tendon was observed alongside the medial condyle of the femur (Fig. 2B-E). The latter appeared to receive the gracilis tendon from the superficial side as well as the semitendinosus tendon from the deep side. Thus, these 3 tendons lost their initial attachments to the perichondrium. The initial pes anserinus was connected with the popliteus muscle sheath (Fig. 2G, H), which provided a fibrous mass at the medial margin (Fig. $2 \mathrm{H}, \mathrm{I}$ ); together with the initial pes anserinus, they wound around the medial half of the tibia. The fascia cruris was still absent from the subcutaneous tissue. On the deep or lateral side of the initial pes, the major part of the SMT was inserted into the popliteus muscle sheath, while the minor part, which appeared to correspond to part of the primitive joint capsule, was attached to the femoral condyle (Fig. 2B). The fibular head was observed in sections containing the pes and SMTs. A notch was not observed on the medial head of the gastrocnemius muscle. These specimens of gestational age 8-9 weeks were not assayed immunohistochemically because of the lack of unstained sections.

\section{Foetuses of gestational age 14-16 weeks}

The knees of all of these specimens were much larger in size than the 8-9-week-old specimens and the condyles of the femur and tibia were well developed. 

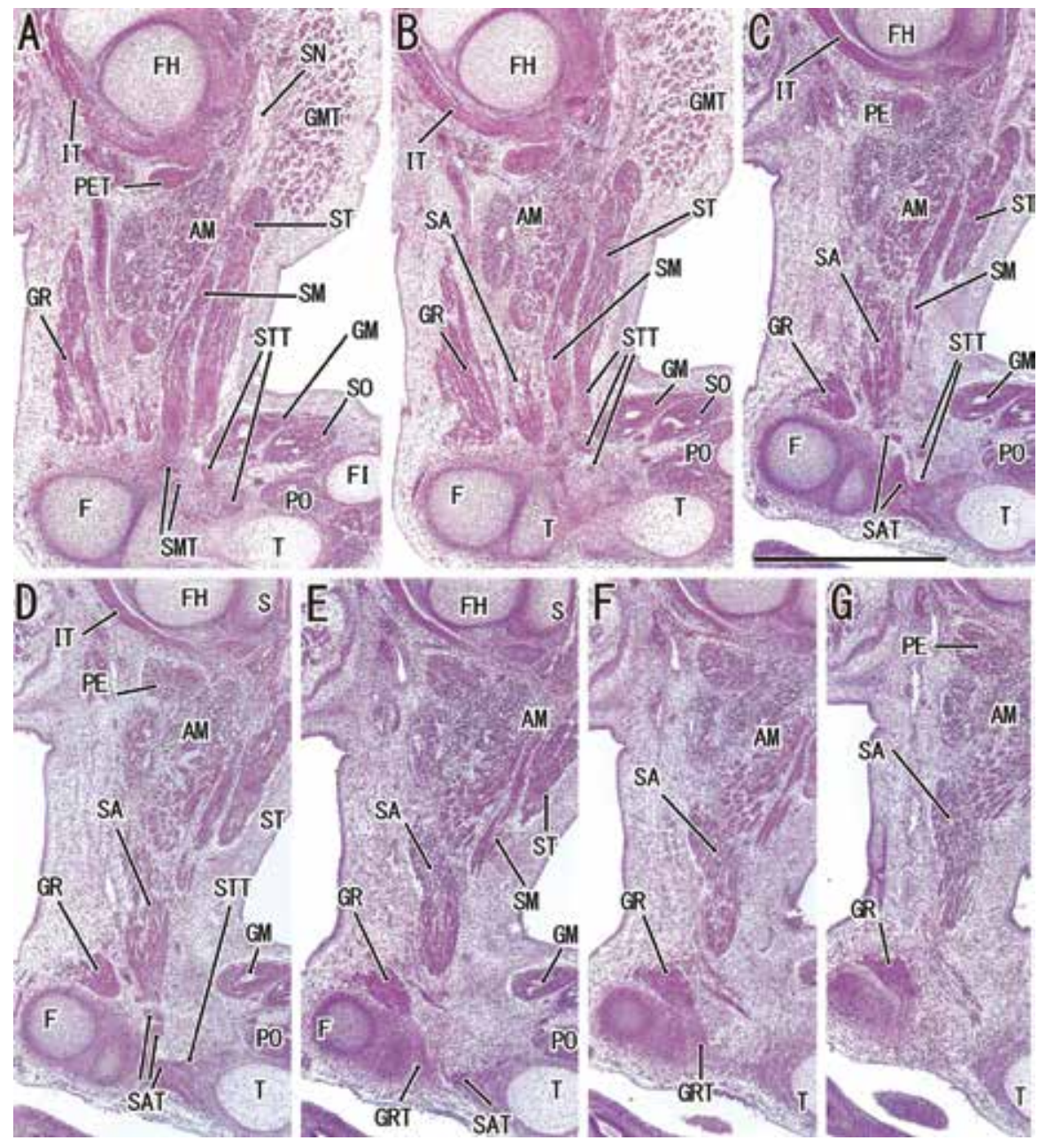

Figure 1. Sagittal sections of a foetal specimen at gestational 8 weeks (CRL $28 \mathrm{~mm}$ ). Panels $\mathbf{A}$ and $\mathbf{G}$ represent the most lateral and medial sites in the figure. All intervals between panels are almost $0.05 \mathrm{~mm}$. Panel $\mathbf{A}$ shows the semitendinosus tendon (STT) and semimembranosus tendon (SMT) inserted into the developing tibia (T) on the proximal side of the popliteus muscle (PO). In panels B-D, the sartorius tendon (SAT) appears to end at a perichondrium of the tibia on the proximal side of the popliteus muscle. Likewise, in panels $\mathbf{E}$ and $\mathbf{F}$, the gracilis tendon (GRT) appears to reach the perichondrium. The fascia cruris is not yet developed. All panels are prepared at the same magnification (scale bar in panel C: $1 \mathrm{~mm}$ ); $\mathrm{AM}$ - adductor magnus muscle; $\mathrm{F}$ - femur; $\mathrm{FH}$ — femoral head; $\mathrm{FI}$ — fibula; $\mathrm{GM}$ - gastrocnemius muscle medial head; GMT — gluteus maximus muscle; GR — gracilis muscle; IT — iliacus tendon; PE — pectineus muscle; PET — pectineus tendon; $\mathrm{S}$ — sciatic bone; SA — sartorius muscle; SM — semimembranosus muscle; SN — sciatic nerve; SO — soleus muscle; ST — semitendinosus muscle.

The fibular head was located on the lateral side of the tibial condyle rather than on the distal side. Thus, the fibular head was not present in sections containing the pes and SMTs, but was located on the far lateral side. The semitendinosus and SMTs crossed the medial head of the gastrocnemius muscle, providing multiple notches on the muscle surfaces (Figs. 3; 4). The SMT divided into a major distal part and a minor proximal part (Figs. 3D; 4C). The major part of the tendon appeared to attach to the tenascin-positive perichondrium of the tibia (Fig. 5), while the minor part covered the external aspect of the joint capsule and reached the femoral condyle. The SMT insertion was located on the immediately medial side of the popliteus muscle.
In contrast to other tendons, the major part of the SMT contained large numbers of CD68-positve macrophages at and near the tibia and popliteus muscle sheath (inserts in Figs. 3; 4). The pes anserinus, which was negative for both versican and tenascin-c (Fig. 5), was continuous with the definite fascia cruris. The fascia was clearly separated from both the perichondrium and the joint capsule. The semitendinosus tendon showed a large U-shaped course around the tibial condyle and popliteus muscle, intersecting with the sartorius tendon on the medial or superficial side. The sartorius tendon ran transversely in its final course (Fig. 3E, F) and, before its terminus, intersected with the gracilis tendon on the lateral or deep side (Fig. 3D-F). 

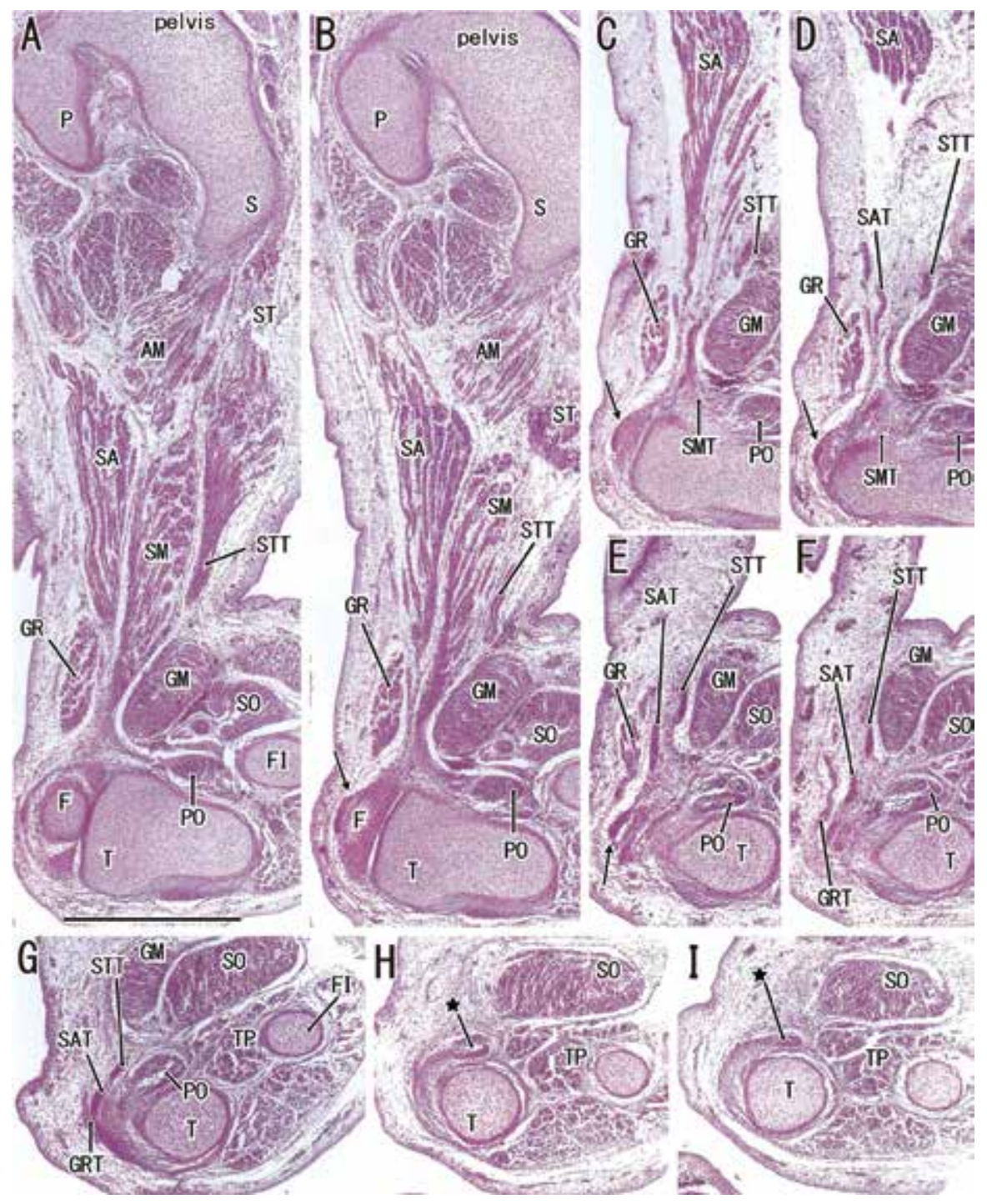

Figure 2. Sagittal sections of a foetal specimen at gestational age 9 weeks (CRL $35 \mathrm{~mm}$ ). Panels $A$ and I represent the most lateral and medial sites in the figure. Intervals between panels are $0.3 \mathrm{~mm}(\mathrm{~A}-\mathrm{B}), 0.1 \mathrm{~mm}(\mathrm{~B}-\mathrm{C}), 0.05 \mathrm{~mm}(\mathrm{C}-\mathrm{D}), 0.2 \mathrm{~mm}(\mathrm{D}-\mathrm{E})$ and $0.05 \mathrm{~mm}(\mathrm{E}-\mathrm{F}, \mathrm{F}-\mathrm{G}, \mathrm{G}-\mathrm{H}, \mathrm{H}-\mathrm{I})$. Panels $\mathbf{A}$ and $\mathbf{B}$ show the semimembranosus tendon (SMT) attached to the proximal end of the popliteus muscle (PO). The arrows in panels B-E indicate fibrous tissue in front of the medial condyle of the femur (F). Panel $\mathbf{F}$ shows that this tissue is continuous with the sartorius tendon (SAT). Panels E-G show the tendons of the pes anserinus (GRT, SAT, STT) joining on the proximal side of the popliteus muscle. Panels $\mathbf{H}$ and I show a connection between the pes and fibrous tissue at the medial margin of the popliteus (star). All panels were prepared at the same magnification (scale bar in panel C: $1 \mathrm{~mm}$ ); AM — adductor magnus muscle; $\mathrm{F}$ — femur; $\mathrm{FI}$ — fibula; $\mathrm{GM}$ - gastrocnemius muscle medial head; $\mathrm{GR}$ - gracilis muscle; GRT — gracilis tendon; $\mathrm{P}$ — pubis; $\mathrm{S}$ — sciatic bone; $\mathrm{SA}$ — sartorius muscle; $\mathrm{SM}$ — semimembranosus muscle; $\mathrm{SO}$ - soleus muscle; ST — semitendinosus muscle; STT — semitendinosus tendon; T — tibia; TP — tibialis posterior muscle.

Thus, these three tendons appeared to form a layered arrangement, with the semitendinosus tendon being deepest, the gracilis tendon being intermediate and the sartorius tendon being most superficial. However, this arrangement was sometimes difficult to detect because the three tendons were arranged almost linearly and parallely (Fig. 4D-F). During the final approach to the medial knee, the gracilis and sartorius tendons took a very tortuous course, with one or both not running along the supero-inferior axis but along the transverse axis. A bursa-like space was seen along the terminal courses of the SMT and semitendinosus tendon. A thick branch of the saphenous nerve passed between the gracilis and sartorius tendons.

\section{DISCUSSION}

Foetal studies suggest that the sites of foetal muscle origin or insertion likely change during development; however, this hypothesis has yet to be widely accepted. The site of insertion of the obturator inter- 


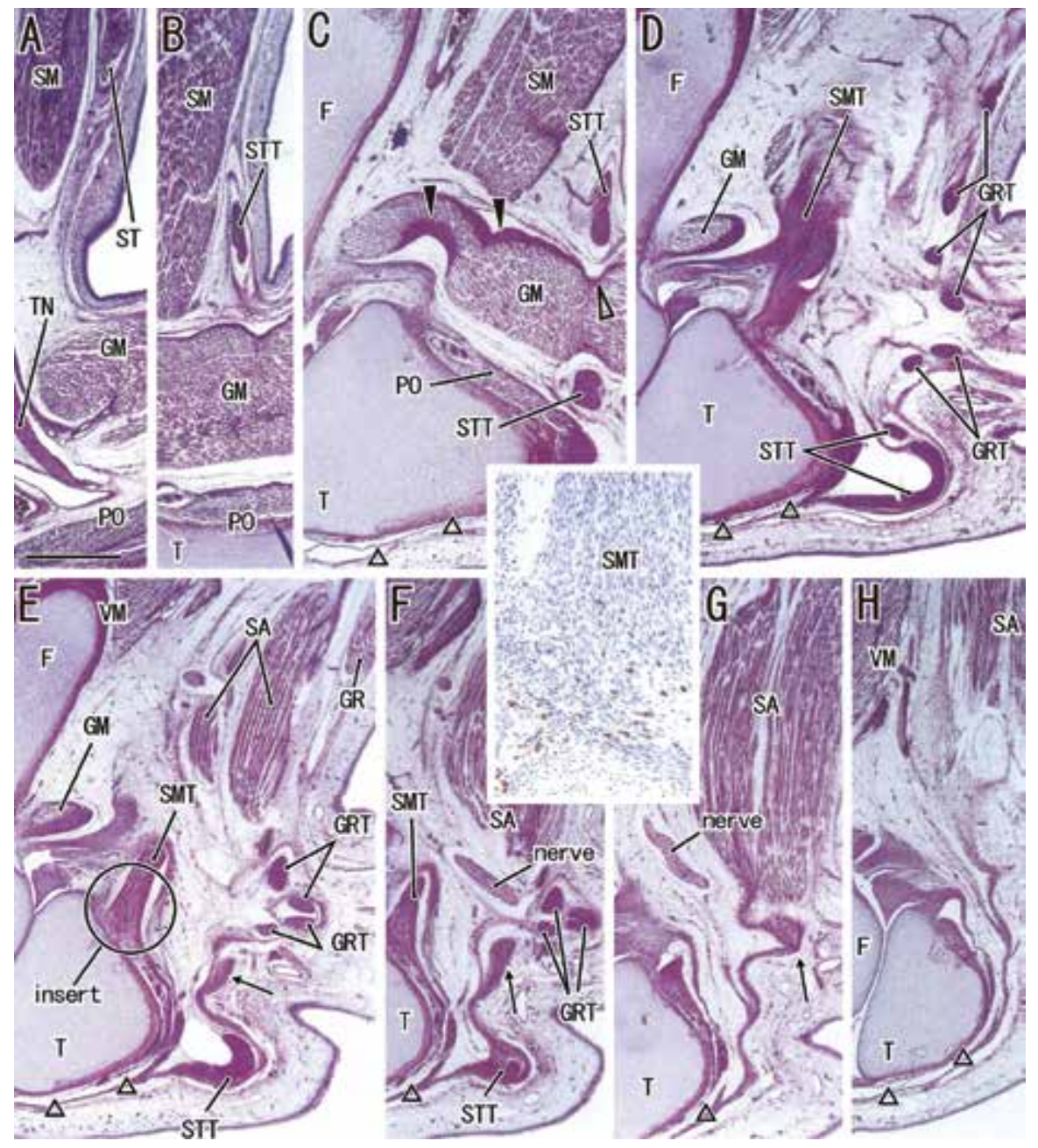

Figure 3. Sagittal sections of a foetal specimen at gestational age 15 weeks (CRL $120 \mathrm{~mm}$ ). Panels $\mathbf{A}$ and $\mathbf{H}$ represent the most lateral and medial sites in the figure. Intervals between panels are $0.8 \mathrm{~mm}(A-B), 0.4 \mathrm{~mm}(B-C, C-D), 0.2 \mathrm{~mm}(D-E)$ and $0.1 \mathrm{~mm}(E-F, F-G, G-H)$. Panel $A$ shows the muscle belly of the semitendinosus muscle (ST), as well as the tibial nerve (TN). Panel B shows the semitendinosus tendon (STT) approaching the knee. Panel C shows notches (arrowheads) on the gastrocnemius medial head (GM) for passage of the semimembranosus tendon (SMT) and semitendinosus tendon (STT). Panels $\mathbf{D}-\mathbf{F}$ show the insertion of the SMT into the tibia, with panel $\mathbf{D}$ also showing this tendon as a thick fibrous bundle inserted into the femur. Panels D-F also show the tortuous courses of the STT and gracilis tendon (GRT). The arrows in Panels E-G indicate the final transverse course of the sartorius tendon (SAT) immediately before joining the GRT. Triangles indicate the fascia cruris. An insert in the centre of the figure, corresponding to the circle in panel $\mathbf{E}$, shows infiltration of CD68-positive macrophages into the SMT. Panels A-H were prepared at the same magnification (scale bar in panel A: $1 \mathrm{~mm}$ ); F — femur; GR — gracilis muscle; PO — popliteus muscle; SA — sartorius muscle; SM — semimembranosus muscle; $\mathrm{T}$ - tibia; $\mathrm{VM}$ — vastus medialis muscle

nus tendon has been reported to change, from the sciatic nerve sheath, via the gluteus medius tendon below the sciatic notch, to the femur trochanteric fossa [13]. Other findings suggest that the insertion of the long tendon of the digastricus muscle posterior belly likely changes from Reichert's cartilage, via the hypoglossal nerve, to the putative anterior belly of the muscle [6]. The brachialis and flexor digitorum profundus muscles carry large attachments to the elbow joint capsule in foetuses, but such attachments are lost after birth [5]. Foetal development of a long muscle is likely to require a definite insertion of a straight tendon, even if the insertion site is changing. Actually, the semitendinosus, gracilis and sartorius tendons were straight during early development, with their later highly tortuous courses apparently reducing mechanical loading greatly. These straight tendons had apparently lost cartilage insertion secondarily, resulting in winding tendons.

It is important to determine the structure or event that forces the pes anserinus tendons to lose their initial site of insertion into the cartilaginous tibia. The present study demonstrated that the pes anserinus tendons, as well as the SMT, were closely related 


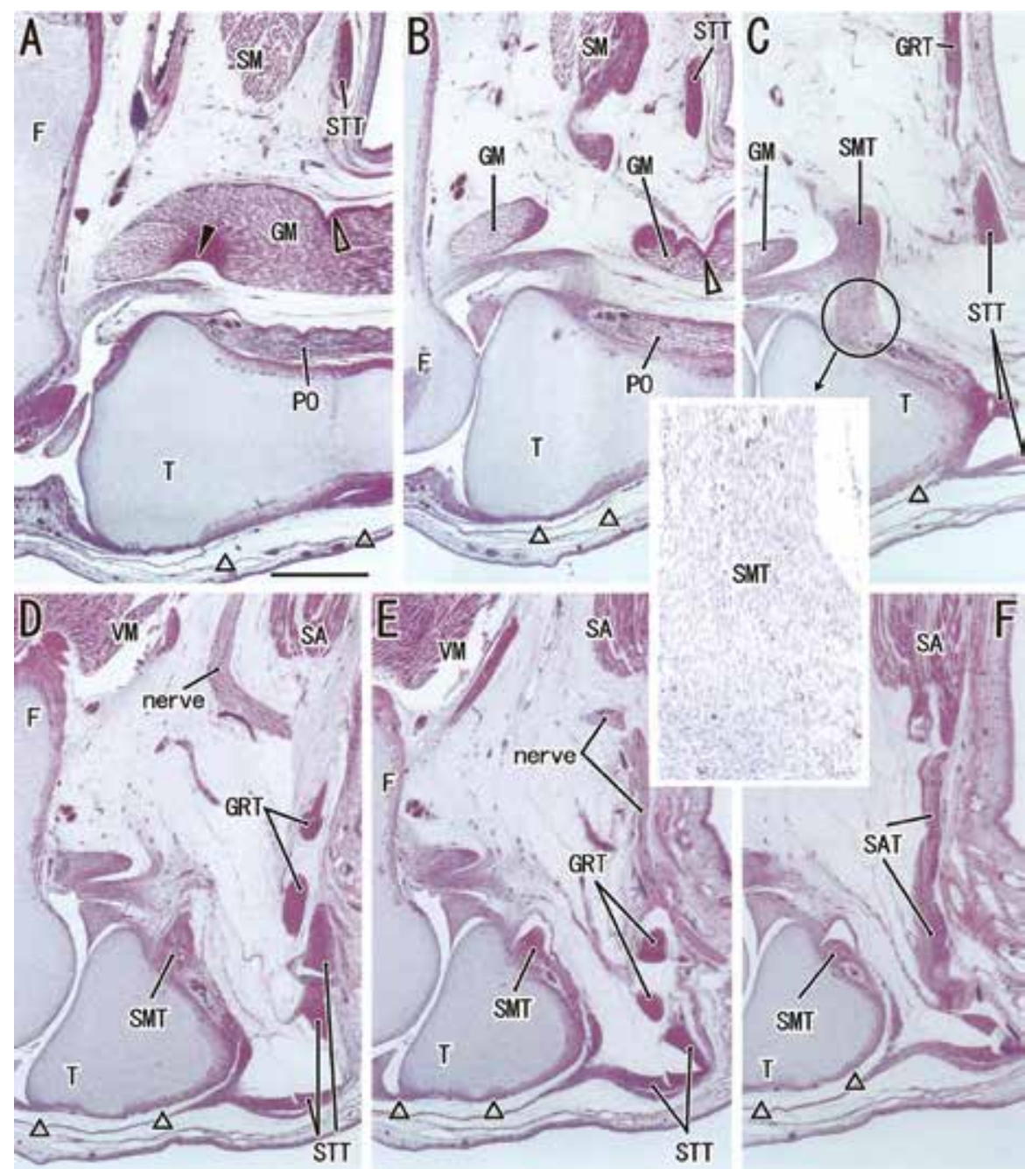

Figure 4. Sagittal sections of a specimen at gestational age 15 weeks (CRL $125 \mathrm{~mm}$ ). Panels $\mathbf{A}$ and $\mathbf{F}$ represent the most lateral and medial sites, respectively, in the figure. Intervals between panels are $0.6 \mathrm{~mm}(A-B)$ and $0.3 \mathrm{~mm}(B-C, C-D, D-E, E-F)$. Panel A shows "notches" on the gastrocnemius medial head (GM) for passage of the semimembranosus tendon (SMT, black arrowhead) and semitendinosus tendon (STT, open arrowhead). Panels A-C show insertion of the SMT into the tibia, while panels B and C show the SMT as a thick fibrous bundle inserted into the femur. Panels D-F show layering of the STT, gracilis tendon (GRT) and sartorius tendon (SAT), with Panel $\mathbf{F}$ showing that the GRT and SAT join initially, later joining the STT outside the figure ( $0.1 \mathrm{~mm}$ medial to panel F). Triangles indicate the fascia cruris. An insert in the centre of the figure, corresponding to the circle in panel $\mathbf{C}$, shows abundant CD68-positive macrophages in the SMT. All panels were prepared at the same magnification (scale bar in panel A: $1 \mathrm{~mm}$ ); $\mathrm{F}$ — femur; PO — popliteus muscle; SA — sartorius muscle; SM — semimembranosus muscle; T — tibia; VM — vastus medialis muscle.

topographically or connected to the popliteus muscle sheath. From gestational age 8-14 weeks, the popliteus muscle extended proximally and medially along the enlarging proximal tibia, with the muscle appearing to push the tendons outside the tibial perichondrium. The fibular head also appeared to migrate proximally. Due to the growing popliteus muscle, tibia and fibula, the topographical anatomy of those 4 tendons changed drastically, especially at 8-9 weeks. In contrast to the pes anserinus tendons, the SMT may still be undergoing reconstruction at
14-16 weeks, as suggested by the large numbers of macrophages at the tendon insertion. During foetal development, CD68-positive macrophages usually accumulate, in association with the re-organisation of muscle and tendon tissues [7]. To compensate for the missing tibial insertion, the SMT seemed to divide, resulting in new insertions into the external lamina of the joint capsule (i.e. the future oblique popliteal ligament) and the popliteus muscle sheath.

The popliteus muscle was likely to "steal" cartilage insertions of those 4 muscles at the medial knee. We 


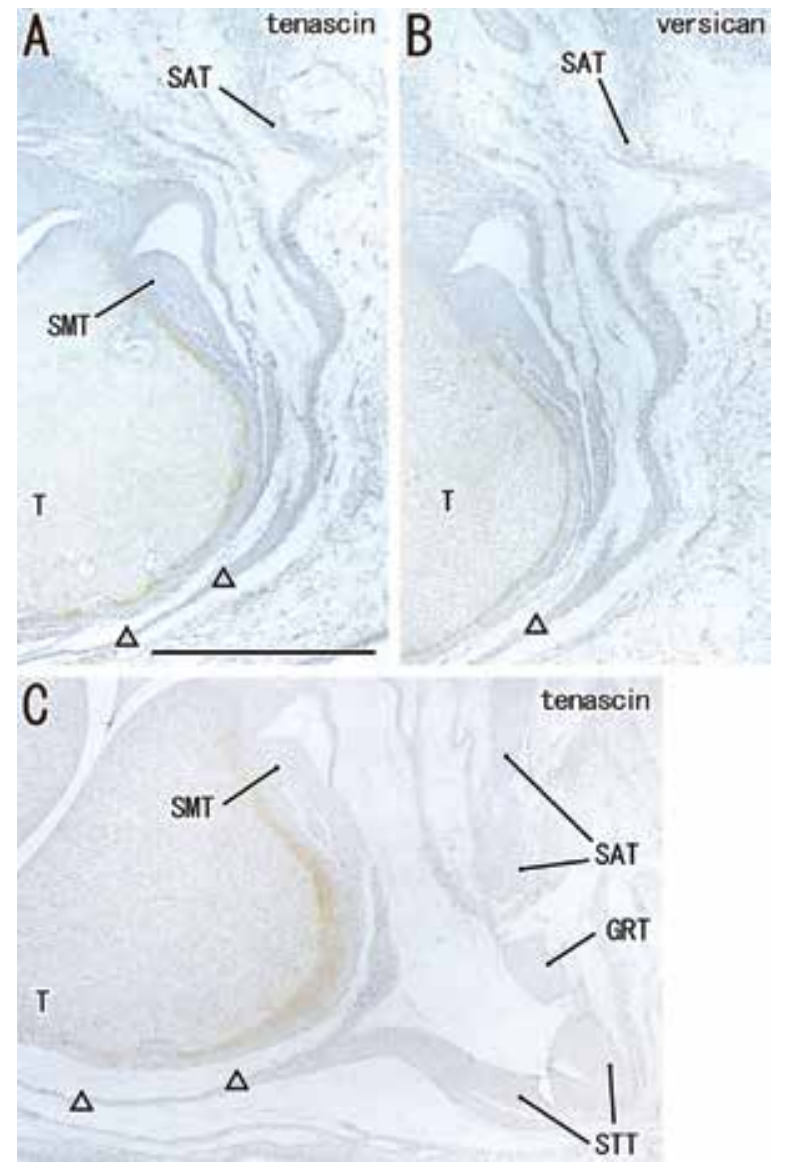

Figure 5. Matrix immunohistochemistry of the pes anserinus and semimembranosus tendon. Sagittal sections. Panels A and B show the binding of anti-tenascin-c and anti-versican antibodies to sections from a specimen in Figure 3. Panel $\mathbf{C}$ shows the binding of anti-tenascin-c antibody to sections from a specimen in Figure 4. The pes anserinus tendons (GRT, SAT, STT) and the semimembranosus tendon (SMT) were negative for both tenascin-c and versican. The tenascin-positive perichondrium of the tibia appears to continue to the insertion of the SMT. Triangles indicate the fascia cruris. All panels were prepared at the same magnification (scale bar in panel A: $1 \mathrm{~mm}$ ); GRT — gracilis tendon; SAT — sartorius tendon; STT — semitendinosus tendon; T — tibia.

speculated a difference in matrix substance between the perichondral and fascial insertions. Versican and tenascin-c are markers of chondrogenesis [19] as well as of the foetal cartilage-tendon interface under tensile stress [10]. Endochondral ossification occurs in versican-rich matrix, whereas perichondral ossification occurs in tenascin-positive fibrous tissues [3]. However, the pes anserinus and SMT were found to be immunohistochemically negative for both versican and tenascin-c, results not directly related to the reduction or absence of mechanical stress on tendinous structures. Although the lack of expression of versican and tenascin-c may have been due to the long-term preservation of these specimens, our finding, that the perichondrium of these specimens was positive, suggests that the pes anserinus did not originate in the perichondrium. The perichondrium was positive for versican and tenascin-c at the site of insertion of the SMT, suggesting that the matrix of the perichondrium was maintained even when tendon insertion sites were changing.

The pes anserinus in adults showed a basic laminar arrangement [11], with the tendons of the semitendinosus, gracilis and sartorius muscles layered in that order from the deep to the superficial side. In foetuses of gestational age 8-9 weeks, however, the gracilis tendon appeared to be located most superficially. Posteromedial tendinous expansion of the semimembranosus and other multiple longitudinal bundles inserting between the pes tendons has been described [11]. Although we did not find such longitudinal fibrous structures in foetuses, this absence may be due to the limitations of histological methods. Variations in pes morphology, such as those reported in adults [4], were likely to occur at gestational age 14-16 weeks, during the reconstruction of highly tortuous tendons. Reconstruction may also occur much later, even after birth, to adjust the topographic relationship of these tendons with nearby bony structures, similar to the experimental shortening of adult tendons [20].

\section{Limitations of the study}

A major limitation of this study was found in a small numbers of specimens (3 specimens) showing the initial stage of the pes tendons inserting to the shaft-like proximal tibia. Likewise, since we did not found the popliteus tendon, we were not able to discuss about a possible relation between the medial or proximal migration of muscle and the tendon development.

\section{CONCLUSIONS}

The pes anserinus tendons (tendons of the semitendinosus, gracilis and sartorius muscles) were straight during early development. However, these tendons had apparently lost cartilage insertion secondarily, resulting in winding tendons in midterm foetuses. Likewise, the semimembranosus tendon changed the insertion from the tibia to the knee joint capsule. The popliteus muscle was likely to "steal" cartilage insertions of these 4 muscles at the medial knee. Reconstruction may occur much later, even 
after birth, to adjust the topographic relationship of these tendons with nearby bony structures. Sites of fetal muscle origin or insertion likely change during development.

\section{REFERENCES}

1. Abe S, Nakamura T, Rodriguez-Vazquez JF Murakami G, Ide $Y$ (2011) Early fetal development of the rotator interval region of the shoulder with special reference to topographical relationships among related tendons and ligaments. Surg Radiol Anat, 33: 609-615.

2. Brent $A E$, Braun T, Tabin CJ (2005) Genetic analysis of interactions between the somatic muscle, cartilage and tendon cell lineages during mouse development. Development, 132: 515-528.

3. Hayashi S, Kim JH, Hwang SE, Shibata S, Fujimiya M, Murakami G, Cho BH (2014) Interface between intramembranous and endochondral ossification in human fetuses. Folia Morphol, 73: 199-205.

4. Ivey M, Prud'homme J (1993) Anatomic variations of the pes anserinus: a cadaver study. Orthopedics, 16: 601-606.

5. Jin ZW, Jin Y, Yamamoto M, Abe H, Murakami G, Yan TF (2016) Oblique cord (chorda obliqua) of the forearm and muscle-associated fibrous tissues at and around the elbow joint: a study of human foetal specimens. Folia Morphol, 75: 493-502.

6. Katori $\mathrm{Y}, \mathrm{Kim} \mathrm{JH}$, Rodriguez-Vazquez JF, Kawase T, Murakami G, Cho BH (2011) Early fetal development of the intermediate tendon of the digastricus and omohyoideus muscles: a critical difference in histogenesis. Clin Anat, 24: 843-852.

7. Kim JH, Abe S, Shibata S, Maki H, Asakawa S, Murakami G, Cho BH (2012) Dense distribution of macrophages in flexor aspects of the hand and foot of mid-term human fetuses. Anat Cell Biol, 45: 259-267.

8. Kjaer M, Langberg $\mathrm{H}$, Heinemeier K, Bayer ML, Hansen M, Holm L, Doessing $S$, Kongsgaard $M$, Krogsgaard MR, Magnusson SP (2009) From mechanical loading to collagen synthesis, structural changes and function in human tendon. Scand J Med Sci Sprts, 19: 500-510.

9. Mackey AL, Heinemeier KM, Koskinen SOA, Kjaer M (2008) Dynamic adaptation of tendon and muscle connective tissue to mechanical loading. Connect Tiss Res, 49: 165-168.

10. Milz S, Benjamin M, Putz R (2005) Molecular parameters indicating adaptation to mechanical stress in fibrous connective tissue. Adv Anat Embryol Cell Biol, 178: 1-71.
11. Mochizuki T, Akita K, Muneta T, Sato T (2004) Pes anserinus: Layered supportive structure on the medial side of the knee. Clin Anat, 17: 50-54.

12. Murchison ND, Price BA, Conner DA, Keene DR, Ollson EN, Tabin CJ, Schweitzer R (2007) Regulation of tendon differentiation by scleraxis distinguishes force-transmitting tendons from muscle-anchoring tendons. Development, 134: 2697-2708.

13. Naito M, Suzuki R, Abe H, Rodriguez-Vazquez JF, Murakami G, Aizawa S (2015) Fetal development of the human obturator internus muscle with special reference to changes in the tendon architecture. Anat Rec, 298: 1282-1293.

14. Nakamura T, Suzuki D, Murakami G, Cho BH, Fujimiya M, Kozuka N (2011) Human fetal anatomy of the posterior semimembranosus complex at the knee with special reference to the gastrocnemio- semimembranosus bursa. Knee, 18: 271-277.

15. Newell RLM, Davies MS (2005) Gray's Anatomy. Editor-In Chief: Standring S. 39th Ed. Elsevier Churchill Livingstone. London, pp. 1461-1470.

16. Nowlan NC, Bourdon C, Dumas G, Tajbakhsh S, Prendergast PJ, Murphy P (2010) Developing bones are differentially affected by compromised skeletal muscle formation. Bone, 46: 1275-1285.

17. Pryce BA, Watson SS, Murchison ND, StaveroskyJA, Dünker N, Schweitzer R (2009) Recruitment and maintenance of tendon progenitors by TGFbeta dignaling are essential for tendon formation. Development, 136: 1351-1361.

18. Rot-Nikcevic I, Reddy T, Dwoning KJ, Belliveau AC, Hallgrímsson B, Hall BK, Kablar B (2006) Myf5-/-:MyoD-/amyogenic fetuses reveal the importance of early contraction and static loading by striated muscle in mouse skeletogenesis. Dev Genes Evol, 216: 1-9.

19. Shibata S, Fukada K, Imai H, Abe T, Yamashita Y (2003) In situ hybridization and immunohistochemistry of versican, aggrecan, and link protein and histochemistry of hyaluronan in the developing mouse limb bud cartilage. J Anat, 203: 425-432.

20. Takahashi M, Ward SR, Marchuk LL, Frank CB, Lieber RL (2010) Asynchronous muscle and tendon adaptation after surgical tensioning procedure. J Bone Joint Surg Am, 92: 664-674.

21. Uchiyama E, Kim JH, Abe H, Cho BH, Rodríguez-Vázquez JF, Murakami G (2014) Fetal development of ligaments around the tarsal bones with special reference to contribution of muscles. Clin Anat, 27: 389-398.

22. Zelzer E, Blitz E, Killian ML, Thomopoulas S (2014) Tendon-to-bone attachment: from development to maturity. Birth Defects Res C Embryo Today, 102: 101-112. 